\title{
Aproximaciones alternativas a la metamorfosis del pulgarcito ${ }^{1}$
}

¿QUÉ SIGNIFICAN LOS ACUERDOS DE PAZ, FIRMADOS el 16 de enero de 1992, en el castillo de Chapultepec entre el gobierno salvadoreño y el FMLN? Caben respuestas distintas y de distinta naturaleza para esta pregunta. Depende del contexto en el que se formula, del enfoque con el que se analiza el proceso y, eventualmente, de los actores a los que se pregunta.

Del contexto. Apretemos el zoom. Imaginémonos que contemplamos El Salvador desde el cielo, que nuestra cámara puede captar todo el flujo de interacciones, percepciones y relaciones que convergen en El Salvador desde todo el mundo. Observaremos, desde la perspectiva de la sociedad internacional, el proceso salvadoreño como un proceso de desarrollo y resolución de un conflicto relevante para su agenda, como un componente de un conflicto regional, en el escenario de la tensión este-oeste, eje estructurador del propio sistema internacional. El recurso a los instrumentos concep- tuales y teóricos de las relaciones internacionales nos permitirá intentar su comprensión y análisis.

Apretemos de nuevo el zoom. Acerquemos la imagen, hasta que El Salvador ocupe todo el espacio. Observemos la realidad nacional. Como en $E l$ cuarteto de Alejandría, la realidad depende de quién la mira, desde dónde se mira, con qué instrumentos teóricos. Podemos así abordar el proceso salvadoreño desde uno y otro lado del conflicto, desde arriba o desde el centro, contemplándolo, respectivamente, como proceso de transición política, como proceso revolucionario y como proceso de paso del estado de naturaleza al contrato social.

Si asumimos el proceso salvadoreño como el que se desarrolla globalmente en distintas fases y dinámicas, a partir de la caída del régimen militar, provocada por el "golpe de los capitanes" del 15 de octubre de 1979, hasta la plena ejecución de los acuerdos de Chapultepec, cuyo análisis supera el planteamiento del presente comentario ${ }^{2}$, cabe con-

1. Este artículo resume las conclusiones y principales tesis del libro del autor La metamorfosis del Pulgarcito. Transición política y proceso de paz en El Salvador, Barcelona, Icaria-FLACSO, 1999.

2. Globalmente, cabe considerar que el proceso salvadoreño se desarrolla en cuatro grandes fases: la que va del "golpe de los capitanes" a la "ofensiva final" del FMLN (enero 1981), que marca abiertamente el estallido de la guerra civil, caracterizada por los intentos de transición y regresión hacia un nuevo régimen; la que va de la "ofensiva final" de 1981 a la de 1989 , que marca el punto de inflexión a partir del que se impone la dinámica negociadora, etapa que se caracteriza por la simultaneidad de guerra civil, transición política, contrainsurgencia y proceso revolucionario; la de la negociación simultánea a la guerra (1989-1992); y la de ejecución de los acuerdos de paz, que implica la instauración de un nuevo régimen democrático, al que se plantea el reto de su consolidación. Una visión detallada del desarrollo del proceso puede obtenerse en la bibliografía señalada. 
templar en su seno varios procesos particulares, cuyos principales rasgos definidores intentaré esbozar para apuntar, finalmente, algunas conclusiones que cabe desprender también del camino analítico recorrido.

(a) La aproximación al proceso salvadoreño en clave internacional implica, por un lado, el análisis del conflicto en cuanto conflicto internacional, y por otro, el de los efectos internos de la dimensión internacional del proceso. Conflicto que, en la perspectiva de una sociedad internacional en un momento determinado de su evolución histórica, se desarrolla en tres niveles relevantes e interrelacionados -nacional, regional e internacional-, y potencialmente conforme a dos dinámicas fundamentales posibles - la de la confrontación o conflicto, y la de la cooperación o negociación-.

La visión y la política de Estados Unidos frente al conflicto resultan determinantes en su desarrollo. Visiones y políticas que se enmarcan en principios orientadores de la política de Estados Unidos hacia América Latina y que se articulan, en primer lugar, partiendo de una implicación especial y de una consideración estratégica previa de Centroamérica, las primeras, en la visión - fundamentalmente desarrollada en el Informe Kissinger- de la crisis centroamericana, desde un encuadramiento este-oeste, planteándose como objetivo la reversión de la revolución en Nicaragua y la necesidad de evitar su triunfo en El Salvador y Guatemala; y, las segundas, a partir de la estrategia de conflicto de baja intensidad, base conceptual de una estrategia, cuyo desarrollo implica, entre otras cosas, la regionalización de la crisis.

En el marco de dicha visión y estrategia, el conflicto salvadoreño es considerado como un "caso test", decisivo en su resultado para el triunfo de la estrategia global, lo que conlleva el diseño de una política frente al conflicto salvadoreño, y una intensa dedicación instrumental a la misma, en el triple ámbito militar, político y socioeconómico, que configura a Estados Unidos como actor determinante y decisivo en la evolución global del conflicto.

Sin embargo, esta política, tanto en el plano regional como en el salvadoreño, entra en un estancamiento doloroso, lo que junto a la emergencia de dinámicas alternativas para la solución del conflicto, por la vía de la cooperación y la negociación, da lugar a un punto de inflexión a partir del cual, tras Esquipulas II, dicha vía se impone como la solución del conflicto, asociándose Estados Unidos progresivamente a la misma.

Frente a Estados Unidos, cabe considerar, en esta dinámica de confrontación o conflicto, al FMLN como el "enemigo" o el actor antagónico fundamental, sin excluir la implicación, en mayor o menor grado, de Nicaragua, Cuba y la Unión Soviética, así como algunos actores no estatales. Frente a éstos, los restantes actores significativos de la sociedad internacional aparecen alineados en torno y a favor de la dinámica de cooperación y negociación.

En definitiva, cabe valorar, desde la perspectiva de Estados Unidos, la evolución del conflicto y su resultado como victoria en los fines y fracaso en los medios. Esto plantea interrogantes acerca de los límites reales entre conflicto nacional e internacional, así como la conveniencia de asumir en su globalidad la problemática interna del conflicto, por parte de los actores internacionales, los cuales por su peso específico se convierten, inevitablemente, en actores internos del mismo.

Por otro lado, para la consideración del desarrollo de la dinámica de cooperación y negociación hacia la solución del conflicto, partimos de la asunción, como factor clave para la imposición de dicha dinámica, de su preferibilidad, en términos de costo-beneficio, a la de la confrontación en la perspectiva de los actores determinantes.

En este sentido, Contadora - proceso de mediación colectiva entre los cinco estados centroamericanos- supone la emergencia de una lectura o visión alternativa de la crisis en el seno de la sociedad internacional, por parte de un grupo significativo de potencias regionales, y de dicha dinámica negociadora como alternativa a su resolución. Aparte del inevitable efecto legitimador de la negociación como alternativa, Contadora dejó tras de sí tal vez un fracaso relativo, pero un triunfo global, al haber posibilitado la contención del conflicto y definido la agenda - si bien priorizando la problemática de seguridad, propia de la preocupación de los actores internacionales- que contiene la temática sustantiva para la solución negociada. Más allá de la crisis centroamericana, cabe contemplar en Contadora la emergencia de una dinámica de búsqueda de soluciones latinoamericanas a los problemas latinoamericanos, que se encuentra en el origen de la construcción de un nuevo orden internacional en América Latina. 
Esquipulas - proceso de negociación directa entre los cinco estados centroamericanos - toma el testimonio de Contadora, y articula en su "Procedimiento" el punto de inflexión de la evolución global del conflicto, imponiendo la dinámica de la negociación y provocando su desescalamiento y solución negociada a nivel regional, basada en determinados principios y en el desplazamiento del centro de gravedad de la seguridad a la política -priorizando así las problemáticas nacionales-, factores determinantes en su negociación y aceptación; centrándose - progresiva erosión de los principios operativos aparte - su ejecución en la problemática nicaragüense, posibilitando tanto la solución de ésta como la del conflicto a nivel regional. No así en El Salvador y Guatemala, para la solución de cuyos conflictos nacionales ofrece un marco y una agenda desacordes con la realidad de los actores y las correlaciones de fuerza que caracterizan el conflicto nacional, cuya solución negociada exige, precisamente, la negociación previa de las reglas del juego de la negociación.

La emergencia y la consolidación de esta dinámica de negociación incluirá y se verá reforzada por la asociación de actores extra-americanos - fundamentalmente, la Unión Europea y Naciones Unidas-, la cual contribuirá a la solución negociada del conflicto en sus diferentes niveles y a la construcción de un nuevo orden internacional en la región.

Cabe señalar que el desmontaje o resolución del conflicto empieza por un nivel o dimensión regional, precisamente por el carácter instrumental de éste respecto a otros niveles, implicando la regionalización estratégica del conflicto la de su solución. Igualmente, la solución en este marco del conflicto nicaragüense, confirma de algún modo la naturaleza fundamentalmente internacional.

Si bien la pacificación regional y los intentos previos de negociación constituyen, entre otros, factores relevantes para la configuración de una dinámica de negociación para la solución del conflicto nacional salvadoreño, será la ofensiva del FMLN en noviembre de 1989 la que constituirá, en la percepción de ambas partes, el punto de inflexión tras el que se impone la dinámica de la negociación a la de la confrontación como vía para la solución del conflicto. A la luz de la experiencia de intentos negociadores previos, precedente en cuanto a la agenda y las posibilidades de marcos negociadores, las partes optan por la me- diación internacional de Naciones Unidas: el Acuerdo de Ginebra y la Agenda de Caracas constituyen el fruto de la negociación de la negociación en que se definen el marco (partes, mediación y entorno), el objetivo y contenido, y el diseño o dinámica del proceso negociador, que cuenta con la decisiva participación de la Sociedad Internacional, a través de la mediación de Naciones Unidas con la coadyuvancia del Grupo de Amigos - fórmula original, novedosa y "sui géneris" de acción internacional-, sin olvidar la aportación de Estados Unidos - de la que no es ajena el calor del fin de la guerra fría-y otros actores del entorno.

Acción internacional en el proceso negociador que ante el desarrollo de éste en una dinámica de descarrilamiento-acuerdo-"resaca", cumple no sólo una función de mediación, impulso y garantía, sino también de reencauzamiento y neutralización del entomo contrario al avance del proceso, configurándose como decisiva para la culminación del mismo.

Si bien cabe contemplar los acuerdos de paz como el guión de una película que los actores están dispuestos a interpretar, y el papel asignado en éste a la Sociedad Internacional como de verificación y aportación de recursos, la realidad de su ejecución dista de reflejar tal automatismo en la interpretación de los actores, pasándose de la negociación de la negociación a la negociación de la ejecución, que se desarrolla conforme a una dinámica de descarrilamiento-negociación/presiónreencauzamiento, resultando fundamental el impulso político de mediación y presión de la Sociedad Internacional —en particular, de Naciones Unidas y el Grupo 4+1 -para la superación de las crisis y el reencauzamiento y culminación exitosa del proceso de ejecución.

En este sentido, cabe señalar que, además del cumplimiento de su mandato verificador e implementador de los acuerdos a través de sus divisiones de derechos humanos, policial, militar y electoral, ONUSAL se constituye de alguna manera en la garantía de la garantía de esa función de impulso político del proceso por parte de la Sociedad Internacional, así como en una experiencia sin precedentes de aportación de la Sociedad Internacional en general y de Naciones Unidas en particular, la primera operación de paz de una nueva generación, que responde al concepto de paz positiva, objeto, más allá de análisis críticos con aportaciones relevantes, de una valoración global positiva. 
Cabe señalar también que constituye un elemento determinante de la positiva intervención de la Sociedad Internacional en el proceso de paz salvadoreño, su asunción de la problemática del conflicto en su globalidad, y no únicamente en perspectiva internacional, promoviendo una solución que contemple respuestas a las preocupaciones de todos los actores relevantes tanto nacionales como internacionales, interrelacionándose las dimensiones interna e internacional del proceso, en el que la internacionalización del conflicto implica la de su solución. Igualmente, el proceso de paz salvadoreño nos muestra que la consecución de la paz negativa pasa por la realización de la paz positiva. Reflejo y a la vez impulso de la evolución de la Sociedad Internacional, cabe extraer de la experiencia pionera y globalmente positiva del proceso de paz salvadoreño algunas lecciones para el perfeccionamiento de los procesos de pacificación de cara al futuro, como la conveniencia de la coherencia y compatibilidad de los diferentes procesos impulsados por la Sociedad Internacional en la sociedad nacional en que se desarrolla el proceso de paz; o la necesidad de aportación no sólo de verificación y recursos a la ejecución, sino también de impulso político; o la conexión entre las diferentes fases del proceso de paz hasta la consolidación de la paz positiva, que implica la asunción del triángulo paz-democracia-desarrollo como objetivo y sentido último del proceso. Presencia de la Sociedad Internacional en el proceso salvadoreño, cuyo reto definitivo consiste en llegar a ser víctima de su propio éxito.

Por último, el análisis del proceso en clave internacional exige no sólo la consideración de éste desde la perspectiva de la Sociedad Internacional, sino también de los efectos internos de su dimensión internacional, de cuyo análisis cabe concluir la naturaleza fundamentalmente interna del conflicto, al tiempo que su internacionalización en la visión y acción de los actores relevantes de la Sociedad Internacional —en especial, de Estados Unidos-, lo que conlleva, por un lado, la conversión de los actores internacionales en actores nacionales o internos, $y$, por otro, la internacionalización de los actores internos al asumir la dimensión internacional del proceso en sus visiones y estrategias, lo que comporta, en el caso del FMLN, su configuración como actor intemacional, con consecuencias tanto en su estructura orgánica y organizativa, como en su acción. Dimensión interna- cional del conflicto determinante a su vez la dimensión internacional de la paz.

(b) Si nos planteamos el análisis del proceso en clave salvadoreña, cabe abordar éste desde tres perspectivas o enfoques teóricos: transicional, revolucionario y contractual.

Desde el primero, partiendo de un enfoque conciliador entre las aproximaciones estratégicas y estructurales, el proceso puede ser contemplado, desde una perspectiva estratégica, como un proceso que se inicia con el derrumbe del régimen anterior, de carácter autoritario, al que sigue un intento de instauración a través de la transición pactada, en cuyo fracaso - determinado fundamentalmente por la insuficiente inclusividad del pacto y la insuficiente capacidad de acatamiento del régimen, así como por la existencia de estrategias y objetivos alternativos para el proceso de cambio político por parte de actores relevantes en los extremos- cabe contemplar el origen de la guerra civil.

En el seno de ésta, frente a la alternativa revolucionaria, asistimos a la evolución de la transición impuesta a la transición negociada, a un proceso de instauración y consolidación progresiva que supone tanto la progresiva aceptación de las reglas del juego político establecidas por el régimen por los actores relevantes, como su progresiva liberalización, hasta la democratización y aceptación definitiva de las reglas que supone la negociación y ejecución de los acuerdos.

Y ello a través de una dinámica cuyas fases vienen marcadas por la suscripción de pactos sucesivos que suponen tanto la progresiva transformación del régimen como la inclusión de actores relevantes.

Régimen cuya coalición fundante guía sus pasos hacia la conquista progresiva de la persistencia estable, tanto en el plano de la eficacia - a través de las reformas socioeconómicas acometidas-, como en el de la legitimidad difusa - a través de los pactos mencionados y de los procesos electorales celebrados-, a pesar de lo cual dista de alcanzar ésta, fundamentalmente por el límite insuperable y distorsionador impuesto por la guerra civil y su dinámica propia.

Desde una perspectiva estructural, por otro lado, cabe contemplar el "cuadrado mágico" definidor del modelo socioeconómico anterior a 1979, como el límite estructural de necesaria superación 
para el establecimiento de una democracia estable en El Salvador; y las reformas socioeconómicas de los gobiernos del PDC, especialmente la agraria, como causa de la superación de dicho límite. Ello, junto a la creación por parte del empresariado de una estructura de intermediación propia competitiva -ARENA - y otros factores, configurará a la guerra, como ya se ha apuntado, en el único y decisivo límite a superar para la democratización plena, posible sólo simultáneamente a una transición negociada de la guerra a la paz que contemple el "desmontaje" del militarismo.

La negociación y ejecución de los acuerdos de paz se configura así al tiempo como la superación de dicho límite y la negociación del "pacto fundacional" de un nuevo régimen político de características poliárquicas y su instauración como solución al conflicto, o cuando menos como alternativa a la canalización de éste por la vía armada.

Sin embargo, cabe señalar en el proceso salvadoreño, contemplado como proceso de transición política, una serie de especificidades que lo diferencian de la tipología de los procesos de transición pactada construida por los transitólogos. Especificidades determinadas en buena medida por la existencia en $\longrightarrow$ junto $a-$ el proceso de transición de otros procesos - simultaneidad y pluralidad que cabría señalar por sí mismas como especificidad fundamental-, entre las que cabría destacar la ausencia de incertidumbre, la transformación de la movilización en rebelión armada ante la represión, el enfrentamiento del pasado, la especificidad del papel de la Fuerza Armada, su dimensión internacional, y la interrelación entre evolución socioeconómica y evolución política, que hace imprescindible la incorporación de consideraciones estructurales - de una perspectiva de contingencia estructurada - para su comprensión $\mathrm{y}$ análisis.

(c) A la luz de las teorías de la revolución, el proceso salvadoreño puede ser contemplado como proceso revolucionario desarrollado en cuatro fases:

* La configuración de una situación prerrevolucionaria en que tiene lugar la construcción del actor, la coalición, el pensamiento y la estrategia revolucionarias.

* La de una situación revolucionaria — caracterizada por la existencia de una soberanía múltiple o doble poder-, en la que tiene lugar un intento de construcción de un orden o régimen revolucionario, hacia dentro -frente a la población y territorios bajo control militar, en los planos de la legitimidad y organización, la eficacia, la movilización de la población, y la capacidad de coerción - y hacia fuera - frente al resto de la sociedad nacional e internacional, tanto en el plano de la legitimidad, a través de la elaboración del discurso o pensamiento revolucionario, como en el de la eficacia o acción revolucionaria, a través del desarrollo de la guerra revolucionaria como eje vertebral de una estrategia integral que contempla diferentes aspectos y escenarios-.

* El cambio en el pensamiento revolucionario redefinido en torno al concepto paradigmático de "revolución democrática", en un proceso que lleva a la afirmación de la poliarquía como objetivo de la revolución.

* La realización de la "revolución democrática" a través de la "revolución negociada", que supone la negociación y ejecución de los acuerdos de paz.

(d) Desde una perspectiva contractual, el proceso en su globalidad puede ser contemplado como el progresivo deterioro del "contrato social A" y el paso, tras el fracaso de la negociación de un nuevo contrato social, a un estado de naturaleza global, en cuyo seno se da el intento simultáneo y confrontado de construcción de un "contrato social B" - el nuevo régimen político-y de un "contra- 
to social C" -el orden revolucionario-, hacia dentro y hacia fuera, a través de su imposición en el campo de juego de la guerra/estado de naturaleza. Así, el proceso de negociación y ejecución de los acuerdos de paz = "contrato social D" implicará el diseño y realización del triple proceso de paso del estado de naturaleza a $\mathrm{D}$ y de $\mathrm{B}$ y de $\mathrm{C}$ a $\mathrm{D}$.

Perspectiva contractual que resalta el carácter fundacional de los acuerdos = contrato social, la existencia de campos de juego simultáneos -para cada actor, el de su contrato social y el del estado de naturaleza global-, y de vasos comunicantes entre éstos, condicionantes de la estrategia de los actores y la evolución del proceso.

Perspectiva contractual desde la que cabe contemplar el proceso como paso de la guerra a la paz. Guerra elemento central en la configuración de la realidad salvadoreña, tanto por su propio carácter central, como en cuanto generadora de mecanismos de sustitución y causa de la permanente distorsión y condicionamiento en el desarrollo global de la realidad política y socioeconómica. Caracterización del proceso como paso de la guerra a la paz con implicaciones decisivas en la negociación y en el diseño y realización de la ejecución de los acuerdos de paz, tanto en la dinámica — paso de la guerra a la negociación como campo de juego principal entre los alternativos; diseño del proceso de ejecución y papel de la Sociedad Internacional- como en el contenido - esencia del do ut des del pacto negociado-.

Consideración de los acuerdos de paz como nuevo contrato social desde la que cabe abordar la dimensión jurídica del proceso, que cabe contemplar, políticamente, en la perspectiva de $\mathrm{C}$, como la construcción de facto en el estado de naturaleza de la capacidad de violencia y legitimidad que confluyen en la ley, poder de guerra base del de negociación que a su vez se traduce en poder legislativo; en la de B, como el paso de la consideración de la guerra como medio para la realización del derecho a fuente del mismo, al reconocer a través de la negociación a $\mathrm{C}$ el poder señalado; y, globalmente, como paso de una situación de ausencia de ley a otra de imperio de la ley y de Estado de derecho. Jurídicamente, sin embargo, nos encontramos ante la transformación de $B$ en $D$ según los procedimientos establecidos legalmente en $\mathbf{B}$, una transición de la ley a la ley. Sin embargo, la especificidad de la transición salvadoreña se traduce en su dimensión jurídica en una serie de particularidades o características únicas, entre las que cabe destacar la juridificación e institucionalización del orden transitorio, la existencia de una doble legitimidad legislativa de facto, la naturaleza metalegislativa y metajurídica de los acuerdos de paz, la configuración de un "bloque de contractualidad" en el ordenamiento jurídico salvadoreño, la especificidad de la transición jurídica en las zonas de control del FMLN, la dimensión jurídica de la Comisión ad hoc para la depuración de la Fuerza Armada y de la Comisión de la Verdad, así como de las elecciones de 1994, y las repercusiones en el funcionamiento del sistema jurídico.

Todo lo cual constituye de hecho a los acuerdos en ordenamiento jurídico transitorio cuyo cumplimiento equivale a una profunda transformación del ordenamiento jurídico, "ley de facto" con vocación y destino de transformación en "ley de iure", en un proceso que jurídicamente concluye con la entrada en vigor del bloque de contractualidad y la nueva legitimidad legislativa nacida de las elecciones de 1994.

(e) Constituye, sin embargo, el punto de llegada del proceso un nuevo régimen político, cuyo análisis permite caracterizarlo como sustancialmente diferente al existente en el punto de partida, y democrático desde una perspectiva poliárquica.

Democracia, sin embargo, en consolidación. Asumiendo una visión amplia o no minimalista de la consolidación democrática, contemplando diferentes ámbitos o direcciones de su desarrollo, puede considerarse el régimen político fundamentalmente consolidado - completada su construcción institucional y la normativa de relación interinstitucional, en "rodaje" práctico esta última-, y el sistema político todavía en construcción y en proceso de consolidación, tanto en el ámbito de las estructuras de intermediación, los partidos y el sistema de partidos, como en el de la cultura política. Proceso de consolidación democrática que plantea a la Sociedad Internacional y a la sociedad salvadoreña el reto de construir una nueva interrelación, superadora de la especificidad o excepcionalidad de la dimensión internacional del proceso salvadoreño.

Más allá de la consolidación democrática, la persistencia estable y la gobernabilidad. El futuro plantea a los salvadoreños el reto de definir y construir, democráticamente, un modelo de nación globalmente viable en lo político, lo social y lo 
económico, partiendo de la experiencia y lecciones del proceso a cuyo conocimiento y comprensión desearía haber contribuido este trabajo.

(f) Por último, cabe apuntar una conclusión intangible, que se desprende de la propia naturaleza de la aproximación acometida: la que se obtiene de haberse aproximado a un mismo objeto o proceso desde diferentes perspectivas o enfoques.

Enfoques y perspectivas todos ellos relevantes, útiles para el conocimiento o iluminación del proceso salvadoreño con luces que no podrían aportar los otros. Procesos simultáneos, cuya explicación en cada enfoque o perspectiva acaba encontrando límites sólo superables recurriendo a su consideración o análisis desde los otros. Procesos en el proceso cuya existencia resulta compatible con la del proceso salvadoreño en cuanto tal. Procesos en el camino, en el durante, y sin embargo en lo esencial una única gran transformación en el antes y el después: la del régimen político. Un antes autoritario y un después democrático. Lo que nos reafirma, en definitiva, la naturaleza fundamental y esencialmente interna de la problemática salvadoreña y su crisis, el carácter de piedra angular o columna vertebral del proceso de transición política - respecto del que los demás adquieren su dimensión relativa y sentido último- en el proceso en su globalidad. Fundamental o vertebral en el qué, explicable sin embargo el cómo sólo en relación o desde la consideración de otros procesos —contractual e internacional-. Proceso de transición que a su vez lo es de paz, o proceso de paz de contenido transicional. Procesos que confluyen porque en el fondo confluyen también en un mismo significado las ideas fuerza o conceptos conductores que constituyen su objetivo último: $\mathrm{paz}=$ democracia $=$ revolución $=$ contrato social .

Pues si bien en una primera aproximación dichas ideas-fuerza o conceptos conductores pueden parecernos diferentes o incluso contradictorios, su profundización y desarrollo puede acabar llevándonos, al alcanzar o traspasar el límite de su potencialidad, del uno al otro. Confluyen unos en otros, y se definen unos a otros. Tal vez porque, en el fondo y más allá, quienes han recurrido y desarrollado tales conceptos lo han hecho en el intento de responder a la pregunta, más esencial, por la sociedad idónea para el hombre y su encuentro consigo mismo y con los otros. Lugar imaginario en el horizonte en que se difuminan y confunden paz, democracia, revolución, contrato social, desarrollo, gobernabilidad ... Reto de su construcción, de desamollo conceptual en positivo, cuya profundización más allá de lo esbozado transciende a su vez el propósito de estas líneas, aunque no el invitar a ello.

\section{Manuel Montobbio}

\section{Bibliografía}

Acevedo, Carlos. "Balance global del proceso de negociación entre el Gobierno y el FMLN", en Estudios Centroamericanos ECA, 519-520, enero-febrero, 1992, pp. 15-53.

Anderson, Thomas. El Salvador. Los sucesos políticos de 1932, 2a. Ed., Costa Rica, Editorial Universitaria Centroamericana, 1982.

Arnson, Cynthia J. El Salvador. A Revolution Confronts the United States, Washington DC, Institute for Policy Studies, 1982.

Arnson, Cynthia J. Crossroads. Congress, the Reagan Administration, and Central America, New York, Pantheon Books, 1989.

Arriola, Joaquín y MENA, David. "Balance global del proceso de negociación entre en gobierno y el FMLN", en Estudios Centroamericanos ECA, 521, marzo, pp. 213-225.

Aya, Rod. "Reconsideración de las teorías de la revolución", en Zona Abierta, 36-37, julio-diciembre, pp. 1-80.

Baloyra, Enrique A. El Salvador en transición, San Salvador: UCA Editores, 1987.

Baloyra, Enrique A. "Salvaging El Salvador" en Journal of Democracy, Vol. 3, 2, abril, 1992, pp. 70-80.

Benítez Manaut, Raúl. La teoría militar y la guerra civil en El Salvador, San Salvador: UCA Editores, 1989.

Benítez Manaut, Raúl. "La ONU y el proceso de paz en El Salvador", en Revista Mexicana de Política Exterior, 34, 1992, pp. 32-52.

Benítez Manaut, Raúl. "Negociaciones de paz en el Tercer Mundo: análisis comparativo", en Revista CIDOB d'Afers Internacionals, 28, 1995, pp. 67-90.

Benítez Manaut, Raúl y Córdova Macías, Ricardo (comps.). México en Centroamérica: expediente de documentos fundamentales, 1979-1986, México, Centro de Investigaciones Interdisciplinarias en $\mathrm{Hu}$ manidades, UNAM, 1989.

Bland, Gary. "Assessing the Transition to Democracy", en Tulchin, Joseph S. con Bland, Gary, 1992, pp. 163-205.

Briones, Carlos y Ramos, Carlos G. La gobernabilidad en Centroamérica: economía, gobernabilidad y democracia en El Salvador, San Salvador, FLACSO, 1995. 
Browning, David. El Salvador. Landscape and Society, Londres, Oxford University Press, 1971. Edición consultada: El Salvador, La tierra y el hombre, 3a. Ed., 1987, San Salvador, Ministerio de Cultura y Comunicaciones.

Córdova Macías, Ricardo y Benítez Manaut, Raúl (Comps.). La paz en Centroamérica: Expediente de documentos fundamentales 1979-1989, México, Centro de Investigaciones Interdisciplinarias en Humanidades, UNAM, 1989.

Córdova Macías, Ricardo. “El Salvador en transición: el proceso de paz", en América Latina hoy, 10, junio, 1995, pp. 63-70.

Cuenca, Breny. El poder intangible. La AID y el Estado salvadorèño en los años ochenta, San Salvador, CRIES-PREIS, 1992.

Ellacuría, Ignacio. "FMLN, el límite insuperable", en Estudios Centroamericanos ECA, 446, noviembre, 1985, pp. 890-897. Cit. de Ellacuría, 1991: 197-208.

Ellacuría, Ignacio. “Análisis ético-político del proceso de diálogo en El Salvador", en Estudios Centroamericanos ECA, 454-455, 1986, pp. 727-751. Cit. de Ellacuría, 1991: 1377-1416.

Ellacuría, Ignacio. "Una nueva fase en el proceso salvadoreño", en Estudios Centroamericanos ECA, 485, 1989, pp. 167-197. Cit. de Ellacuría, 1991: 18551897.

Ellacuría, Ignacio. Veinte años de historia en El Salvador (1969-1989). Escritos políticos 3 Vols., San Salvador: UCA Editores, 1991.

FLACSO. Programa El Salvador. El proceso electoral 1994, San Salvador, FLACSO, 1995.

Gordon, Sara. Crisis política y guerra en El Salvador, México, Siglo XXI Editores, 1989.

Guido Béjar, Rafael. "El Salvador: ¿una democracia diferente? (Apuntes para una definición del régimen político salvadoreño)", en Guido Béjar, Rafael y Roggenbuck, Stefan (Eds.), 1995: 17-41.

Guido Béjar, Rafael y Roggenbuck, Stefan (Eds.). El Salvador a fin de siglo, San Salvador, Fundación Konrad Adenauer-UCA, 1995a.

Guido Béjar, Rafael y Roggenbuck, Stefan (Eds.). Sociedad participativa en El Salvador, San Salvador, Fundación Konrad Adenauer- UCA, $1995 b$.

Guido Béjar, Rafael y Roggenbuck, Stefan (Eds.). Partidos y actores políticos en transición. La derecha, la izquierda y el centro en El Salvador, San Salvador, Fundación Konrad Adenauer-UCA, 1996.

Guidos Béjar, Rafael. El ascenso del militarismo en El Salvador, 4a. Ed., San Salvador: UCA Editores, 1988.

Holiday, David y Stanley, William. "Building the Peace: Preliminary Lessons from El Salvador", en Journal of International Affairs, 46, 1993, pp. 415-438.
Holiday, David y Stanley, William. "En la mejor de las circunstancias: ONUSAL y los desafíos de la verificación y el fortalecimiento en El Salvador", en Estudios Centroamericanos ECA, 584, junio, 1997, pp. 549-573.

Karl, Terry Lynn. "Imposing Consent? Electoralism vs. Democratization in El Salvador", en Drake, Paul W. y Silva, Eduardo (Eds.), Elections and Democratization in Latin America, 1980-85, San Diego, University of California, Center for US-Mexican Studies and Institute of the Americas, 1986.

Karl, Terry Lynn. "Exporting Democracy: The Unanticipated Effects of U.S. Electoral Policy in El Salvador", en Hamilton, Nora; Frieden, Jeffry A.; Fuller, Linda y Pastor, Manuel Jr. (Eds.), 1988.

Karl, Terry Lynn. "El Salvador's negotiated revolution" en Foreign Affairs, 71, 1992, pp. 147-164.

Karl, Terry Lynn. "The hybrid regimes of Central America", en Journal of Democracy, Vol. 6, 3, julio, 1995, pp. 72-86.

Lungo, Mario. El Salvador en los 80: contrainsurgencia y revolución, San Salvador: Editorial Universitaria, 1991.

Montgomery, Tommie Sue. Revolution in El Salvador. From civil strife to civil peace, 2a. Ed., Boulder, Westview Press, 1995a.

Montgomery, Tommie Sue. "Getting to Peace in El Salvador: The Roles of the United Nations Secretariat and ONUSAL", en Journal of Interamerican Studies and World Affairs, Vol. 37, 4, 1995b, pp. 139-172.

Montobbio, Manuel. "Construcción nacional y crisis en El Salvador: una aproximación contractual a la problemática salvadoreña", en Tiempo de Paz, 24-25, 1992, pp. 25-31.

Montobbio, Manuel. "La crisis centroamericana y la construcción de un nuevo orden internacional en América Latina", en Revista CIDOB d'Afers Internacionals, 37, 1997, pp. 131-149.

Montobbio, Manuel. La metamorfosis del Pulgarcito. Transición política y proceso de paz en El Salvador, Barcelona: Icaria-FLACSO, 1998.

Naciones Unidas. Las Naciones Unidas y El Salvador 1990-1995, Nueva York, Naciones Unidas, 1995.

Paramio, Ludolfo. "La revolución como problema teórico", en Revista del Centro de Estudios Constitucionales, 7, septiembre-diciembre, 1990, pp. 151-174.

Rosa, Herman. $\dot{A} D$ y las transformaciones globales en El Salvador. El papel de la asistencia económica de los Estados Unidos desde 1980, Managua, CRIES, 1993.

Rouquié, Alain. Guerras y paz en Centroamérica, México: Fondo de Cultura Económica, 1994. 
Seligson, Mitchell A. y Córdova Macías, Ricardo. Perspectivas para una democracia estable en El Salvador, San Salvador: IDELA, 1992.

Seligson, Mitchell A. y Córdova Macías, Ricardo. El Salvador. De la guerra a la paz. Una cultura política en transición, San Salvador: IDELA-University of Pittsburg-Fundaungo, 1995.

Soto, Álvaro de y Castillo, Graciana del. "Obstacles to peacebuilding”, en Foreign Policy, 94, 1994, pp. 69-83.

Stahler-Sholk, Richard. "El Salvador's Negotiated Transition: From Low-Intensity Conflict to Low-Intensity Democracy", en Journal or Interamerican
Studies and World Affairs, Vol. 36, 4, 1994, pp. 159.

Tulchin, Joseph S. y Bland, Gary (Eds.). Is there a Transition to Democracy in El Salvador?, Washington DC, Woodrow Wilson, 1992.

Vilas, Carlos. "Un balance de la ejecución de los Acuerdos de Paz en El Salvador", en Papers. Revista de Sociologia, 49, Universidad Autónoma de Barcelona, 1996, pp. 77-94.

Walter, Knut y Williams, Philip J. "The Military and Democratization in El Salvador", en Journal of Interamerican Studies and World Affairs, Vol. 35, 1, 1993, pp. 39-88. 